\title{
Foreword: the year at Pharmacogenomics
}

\author{
Sarah Jones*,1 \\ ${ }^{1}$ Commissioning Editor, Pharmacogenomics, Future Medicine \\ *Author for correspondence: s.jones@futuremedicine.com
}

I would like to wish all our readers a happy new year and to introduce you to the $20^{\text {th }}$ volume of Pharmacogenomics. Looking back over 2018, our most read research article was a study of patient perceptions and utilization of pharmacogenomics (PGx) testing in an integrated community health system by Lemke $e$ t al. [1]. The paper reported that patients valued pharmacogenetics testing and highlighted the role patient experiences, understanding and result utilization will play in its implementation.

Pharmacogenomics published the paper "Deep learning in pharmacogenomics: from gene regulation to patient stratification" by Kalinin et al. in May 2018 [2], which sparked interest on social media sites. This perspective looked at examples of current and future applications of deep learning in PGx and predicted that it will be widely used to predict personalized drug response and optimize medication selection and dosing in the future. We are pleased to be publishing a further paper by the group in February 2019 entitled "Pharmacogenomics in the age of GWAS, omics atlases, and PheWAS."

Another paper generating interest was a review of standardisation and the adoption of pharmacogenomics published in July by Caudle et al. [3] The paper looked at how lack of standardized approaches and terminology in clinical laboratory processes impede workflow and reviewed resources documenting nomenclature for PGx alleles and variants.

Going forward, the editorial team are looking forward to the new year, with continued publication of the latest cutting-edge research, as well as insightful and topical reviews and perspective papers.

We appreciate all feedback from the pharmacogenomics community regarding the direction of our content, especially suggestions of any priority topics in the field that you feel the journal should cover. We welcome unsolicited research, review and opinion article proposals, amongst others, and would be delighted to hear from you if you are interested in submitting to the journal.

We also encourage you to follow the Pharmacogenomics Twitter account (@pgsjournal) to keep up to date with journal content and PGx news.

I would finally like to take this opportunity to thank our readers, contributing authors, valued Editorial Board members and peer reviewers for their continued support and collaboration as we continue to publish the latest high-impact research. We very much look forward to working with you all over the next year.

\section{References}

1. Lemke AA, Hulick PJ, Wake DT et al. Patient perspectives following pharmacogenomics results disclosure in an integrated health system. Pharmacogenomics 19(4), 321-331.

2. Kalinin AA, Higgins GA, Reamaroon $\mathrm{N}$ et al. Deep learning in pharmacogenomics: from gene regulation to patient stratification. Pharmacogenomics 19(7), 629-650 (2018).

3. Caudle KE, Keeling NJ, Klein TE, Whirl-Carrillo M, Pratt VM, Hoffman JM. Standardization can accelerate the adoption of pharmacogenomics: current status and the path forward. Pharmacogenomics 19(10), 847-860 (2018). 
